Revue internationale P.M.E.

Économie et gestion de la petite et moyenne entreprise

\title{
Les alliances stratégiques asymétriques entre multinationales et PME
}

\author{
Le cas de Danone - Djurdjura en Algérie
}

Foued Cheriet, Frédéric Le Roy et Jean-Louis Rastoin

Volume 21, numéro 1, 2008

URI : https://id.erudit.org/iderudit/038002ar

DOI : https://doi.org/10.7202/038002ar

Aller au sommaire du numéro

Éditeur(s)

Presses de l'Université du Québec

ISSN

0776-5436 (imprimé)

1918-9699 (numérique)

Découvrir la revue

Citer cet article

Cheriet, F., Le Roy, F. \& Rastoin, J.-L. (2008). Les alliances stratégiques asymétriques entre multinationales et PME : le cas de Danone - Djurdjura en Algérie. Revue internationale P.M.E., 21(1), 35-68.

https://doi.org/10.7202/038002ar
Résumé de l'article

L'objet de ce travail est d'analyser un cas d'alliance stratégique entre une firme multinationale (Danone) et une PME agroalimentaire (Djurdjura) en Algérie. Des situations d'asymétrie de taille, de pouvoir de négociation et de contrôle font de cet accord une figure spécifique des relations interentreprises. Les effets de l'accord ont été analysés sur trois plans : l'entité elle-même, les autres filiales du groupe algérien et le secteur des produits laitiers frais en Algérie. Le cadre d'analyse mobilisé combine l'analyse stratégique et une approche par l'apprentissage organisationnel. Les résultats obtenus attestent d'une prudence de la PME face aux manoeuvres de la FMN pour freiner son apprentissage et accroître son contrôle. 


\title{
Les alliances stratégiques asymétriques entre multinationales et PME \\ Le cas de Danone - Djurdjura en Algérie ${ }^{1}$
}

Foued CHERIET

École supérieure d'agronomie de Montpellier

Frédéric LE ROY

Université de Montpellier I

Jean-Louis RASTOIN

École supérieure d'agronomie de Montpellier

\section{MOTS CLÉS}

\section{Asymétrie - Alliance stratégique - Apprentissage PME - FMN - Pays émergent}

\begin{abstract}
LES AUTEURS
FOUED CHERIET est allocataire de recherche à l'unité mixte de recherche Moisa « Marchés, organisations, institutions et stratégies d'acteurs » de l'École supérieure d'agronomie de Montpellier. II prépare une thèse de doctorat en management stratégique portant sur l'instabilité des alliances asymétriques entre firmes multinationales et entreprises agroalimentaires en Méditerranée. Ses intérêts de recherche portent sur les conflits dans les relations interorganisationnelles, les stratégies des firmes multinationales et les investissements directs à l'étranger.

FRÉDÉRIC LE ROY est professeur à l'Université de Montpellier I (ISEM) et au Groupe supérieur de commerce de Montpellier. Directeur de l'ERFI et codirecteur de l'ISEM, il a publié de nombreux articles scientifiques et plusieurs ouvrages. Ses recherches actuelles portent essentiellement sur l'analyse des relations de concurrence et de coopération. II a publié notamment, en 1999, Stratégie militaire et management stratégique des entreprises, aux éditions Economica, en 2002, La concurrence, aux éditions Vuibert et en 2007, Stratégies collectives, avec Saïd Yami.
\end{abstract}

1. Nous remercions les lecteurs anonymes du comité scientifique de la $\mathrm{II}^{\mathrm{e}}$ Conférence internationale du CEMED de l'École de management de Marseille ainsi que les trois évaluateurs anonymes de la Revue internationale PME pour leurs remarques. 
JEAN-LOUIS RASTOIN est ingénieur agronome. Titulaire d'un doctorat d'État ès sciences économiques et agrégé de sciences de gestion, il est actuellement professeur d'économie et gestion des entreprises à l'École supérieure d'agronomie de Montpellier et directeur de l'unité mixte de recherche Moisa «Marchés, organisations, institutions et stratégies d'acteurs ». Son enseignement et ses recherches portent sur la prospective des systèmes agroalimentaires et les stratégies d'entreprises. Auteur de plus de 150 publications scientifiques, il a présidé durant six ans l'Association internationale d'économie alimentaire et agroindustrielle (AIEA2), dont il est aujourd'hui vice-président. II est correspondant national de l'Académie d'agriculture de France.

\title{
RÉSUMÉ
}

L'objet de ce travail est d'analyser un cas d'alliance stratégique entre une firme multinationale (Danone) et une PME agroalimentaire (Djurdjura) en Algérie. Des situations d'asymétrie de taille, de pouvoir de négociation et de contrôle font de cet accord une figure spécifique des relations interentreprises. Les effets de l'accord ont été analysés sur trois plans: l'entité elle-même, les autres filiales du groupe algérien et le secteur des produits laitiers frais en Algérie. Le cadre d'analyse mobilisé combine l'analyse stratégique et une approche par l'apprentissage organisationnel. Les résultats obtenus attestent d'une prudence de la PME face aux manœuvres de la FMN pour freiner son apprentissage et accroître son contrôle.

\begin{abstract}
The aim of this work is to study a case of strategic alliance between a multinational firm (Danone) and an agrifood SME (Djurdjura) in Algeria. Situations of size, bargaining power and control asymmetries turn this agreement into a specific case of inter-firms relations. The consequences of the agreement were analysed on three levels: the entity itself, other subsidiary companies of the Algerian group and the fresh dairy sector in Algeria. The conceptual framework combines strategic analysis and an organisational learning approach. The results show prudence from the SME in relation to the operations of the FMN in order to slow down its learning and to increase its control.
\end{abstract}

\section{RESUMEN}

El objetivo de este trabajo es analizar un caso de alianza estratégica entre una firma multinacional (Danone) y una pequeña y mediana empresa (PyME) (Djurdjura) en el sector agro-alimenticio en Argelia. Por sus asimetrías de tamaño, de poder y de negociación, este acuerdo es un caso específico de relaciones entre empresas. Las consecuencias del acuerdo fueron analizadas en tres niveles : la entidad en si, las otras filiales del grupo argelino y el sector de productos frescos en Argelia. El enfoque teórico utilizado combina análisis estratégico y análisis por el aprendizaje organizacional. Los resultados obtenidos muestran que las PyMEs son prudentes frente a las acciones de la firma multinacional para frenar su aprendizaje y aumentar su control. 


\section{ZUSAMMENFASSUNG}

Das Ziel dieser Arbeit ist die Untersuchung einer strategischen Allianz zwischen einem multinationalen Unternehmen (Danone) und einem algerischen kleinen und mittleren Unternehmen aus dem Lebensmittelsektor (Djurdjura). Unterschiede in der Unternehmensgrösse, der Verhandlungsstärke und der Kontrollmacht charakterisieren diese Partnerschaft. Die Auswirkungen des Abkommens wurden auf drei verschiedenen Niveaus gemessen und zwar im Hinblick auf die strategische Allianz selbst, auf andere Tochterfirmen des algerischen Unternehmens und auf den gesamten algerischen Markt für Frischmilchprodukte. Die durchgeführte Untersuchung verbindet eine strategische Analyse und den Ansatz der lernenden Organisation. Die Resultate der Studie belegen die Vorsicht der KMU gegenüber den Vorhaben des multinationalen Unternehmens, den Lernprozess zu verzögern und die Kontrolle zu vergrössern.

\section{Introduction}

Si l'arrivée d'une multinationale dans un pays en transition peut déboucher sur une amélioration de la compétitivité des petites et moyennes entreprises locales et contribuer à une restructuration industrielle, elle suscite également des inquiétudes quant au devenir de ces entreprises et de l'emploi. Un tel évènement est aussi perçu comme une volonté de l'État de se désengager du secteur privé, ce qui ravive la question de souveraineté économique nationale. Plusieurs auteurs (dont Ohamé, 1990) soutiennent que l'arrivée d'entreprises étrangères exerce un effet positif sur le tissu industriel national. $\mathrm{Au}$ contact de ces entreprises, les PME locales pourraient bénéficier d'un apport considérable en matière de gestion et de savoir-faire technologique.

Ce débat conduit aux interrogations suivantes: est-ce que la faible taille d'une PME crée une dépendance à l'égard de la firme multinationale (FMN)? Est-ce, au contraire, une opportunité d'apprentissage organisationnel pour la PME? Comment la PME peut-elle gérer cette relation asymétrique? Doit-elle s'acheminer vers une relation de type hiérarchique à l'égard de la FMN? Comment la PME peut-elle compenser le «handicap» de sa petite taille et de son faible pouvoir de négociation? Qu'est-ce qui - dans une situation d'alliance stratégique asymétrique - fait la force du «faible»? La question du «choix» du recours de la PME à une alliance stratégique avec une FMN mérite aussi d'être examinée (Fernandez et Noel,1993). En d'autres termes, notre question centrale peut s'exprimer ainsi: Quels sont les enjeux pour une PME engagée dans une alliance stratégique avec une FMN dans le contexte d'un pays en développement? 
Ces enjeux sont liés à la pertinence du choix du partenaire et au processus de négociation de l'accord par rapport aux motivations stratégiques de départ et aux résultats escomptés pour l'entité commune, le groupe local d'appartenance et la compétitivité dans le secteur d'activité.

Une littérature conséquente a tenté de comparer la performance relative des alliances stratégiques comme mode d'entrée des FMN (Gomes-Casseres, 1987 ; Li, 1995) avec celle des autres modes (filiales, accords, représentation commerciale, etc.), concluant le plus souvent à des risques d'instabilité moins élevés pour les filiales autonomes (Bleeke et Ernst, 1991; Kent,1991). D'autres études se sont explicitement intéressées aux alliances stratégiques dans les PVD (Lee et Beamish, 1995; Sim et Yunus, 1998; Gill et Butler, 2003) ou dans les pays en transition (Artisien et Buckley, 1985; Steensma et Lyles, 2000). Enfin, de rares contributions ont abordé les relations entre FMN et PME en signalant que les modèles d'analyse appliqués aux FMN ne peuvent être identiques à ceux examinant les relations entre PME (Tallman et Shenkar, 1994, O’Dwyer et O'Flynn, 2005).

Afin de suggérer des éléments de réponse à ces questions, ce travail se propose d'examiner les trois questions suivantes à travers l'analyse d'une expérience d'alliance stratégique asymétrique entre une firme multinationale et une PME d'un pays en transition, l'Algérie.

Q1: Quelles ont été les motivations stratégiques du partenaire local ayant conduit au choix du partenaire étranger et à ce type d'accord?

Q2: Comment se sont déroulées les négociations et quels en sont les résultats en termes de contrôle et d'organisation de la PME locale?

Q3: Quels sont les principaux résultats de l'accord $a$ ) sur la PME locale, $b$ ) sur ses relations avec son groupe d'appartenance et $c$ ) sur la concentration-concurrence du secteur d'activité ?

Ces questions sont traitées par l'étude d'un cas concret d'alliance stratégique entre Danone (groupe multinational) et Djurdjura (PME algérienne). Nous présenterons dans un premier temps les fondements théoriques de notre analyse des alliances stratégiques asymétriques, puis nous exposerons dans la partie empirique la méthodologie adoptée (étude de cas) suivie de la discussion de nos résultats. 


\section{Référentiel théorique: les alliances stratégiques entre PME et FMN}

Nous ferons, dans un premier temps, une recension de la littérature consacrée aux alliances, puis nous traiterons de l'asymétrie des partenaires.

\subsection{Alliances stratégiques: une profusion de définitions et une multitude d'approches}

Il existe dans le cadre des relations contractuelles et conventionnelles entre les entreprises une profusion de termes et d'appellations qui désignent autant de modalités d'accords (Baudry, 1997). Ainsi, «les nouvelles formes de coopération » entre les entreprises font appel à une très grande diversité d'appellations.

De manière générale, nous pouvons nous rapporter à la définition suivante pour caractériser les alliances stratégiques: «une alliance interentreprises est un lien tissé volontairement entre plusieurs firmes souveraines, restant indépendantes en dehors de ce lien. Elle se caractérise par la mise en commun, par aux moins deux entreprises, d'une fraction de leurs ressources pour la poursuite d'objectifs conjoints dans un espace donné et l'obtention d'avantages réciproques » (Jolly, 2001, p. 17).

Cette définition met l'accent sur l'autonomie des deux firmes partenaires et le partage des responsabilités et des résultats. Certains auteurs distinguent en outre deux types d'alliances: le premier, où il y a création d'entité juridique nouvelle avec un partage de capital, et le second, pour lequel la convention de coopération est sans structure sociétaire ni lien capitalistique (il s'agit, par exemple, des échanges de licence [Hennart, 1988]). Même si elles font partie des alliances stratégiques, les joint ventures sont souvent traitées de manière particulière et sont assimilées à des engagements à plus long terme (Pfeffer et Novak, 1976).

Dans ce travail d'évaluation empirique, l'alliance stratégique sera définie comme une relation contractuelle entre deux entités juridiques indépendantes qui partageront formellement les responsabilités et le pouvoir de décision en vue de la réalisation d'objectifs déterminés.

Certains auteurs distinguent la simple alliance de celle dont le caractère est stratégique: "une alliance est stratégique lorsqu'elle associe deux ou plusieurs entreprises concurrentes ou potentiellement concurrentes et que les ressources mises en commun et les objectifs visés par l'entente de collaboration touchent à des secteurs clés ou à des activités vitales. L'entente de collaboration se concrétise par un engagement quasi irréversible de la part des alliés» (Garette et Dussauge, 1990). 


\subsection{PME / FMN : asymétries de pouvoir et de taille}

Si de nombreuses études ont été consacrées à l'analyse des alliances stratégiques entre grandes firmes multinationales, peu ont traité des partenariats entre FMN et PME dans les économies développées et les travaux consacrés aux alliances entre FMN et PME des pays en voie de développement (PVD) sont quasi inexistants. Pourtant, le phénomène ne cesse de prendre de l'ampleur tant en ce qui concerne la quantité que la diversité des formes d'alliance. Dans un rapport de l'OCDE (2001) cité par Tahi et Belahcen (2000), il est noté un accroissement des alliances stratégiques entre FMN et PME qui passent de 290 opérations en 1996 à 453 en 1999. Au-delà de cet aspect quantitatif, les relations entre FMN et PME posent le problème d'une double asymétrie, de taille et de pouvoir.

En effet, même si certains auteurs lient le pouvoir de négociation non pas à la taille des firmes mais plutôt à l'évaluation des actifs apportés (Yan, 1998), la taille du partenaire peut constituer un atout certain lors de la discussion de l'accord. Trois points de vue s'affrontent concernant la stabilité de l'alliance et l'équilibre entre les partenaires. D'après Killing (cité par Blodgett, 1992), la stabilité de l'alliance est positivement liée à l'existence d'un partenaire dominant exerçant une influence sur l'autre. Pour une plus grande stabilité de l'alliance, ce déséquilibre devrait être en faveur du partenaire dominant étranger (Yan et Gray, 1994; Geringer et Hébert, 1991) ou du partenaire local (Artisien et Buckley, 1985; Park et Ungson, 1997). Inversement, de nombreuses études ont montré le caractère déterminant d'un équilibre dans les rapports et les relations entre partenaires pour les performances de l'alliance (Garette, 1989; Blodgett, 1992).

L'alliance stratégique est liée dans la littérature à la notion de dépendance. Cette dernière est accentuée par la spécificité des actifs et l'asymétrie d'information entre les partenaires (Buckley et Casson, 1996) et crée une relation quasi irréversible entre les alliés. Cette dépendance amoindrit le pouvoir au sein de l'alliance et condamne le partenaire dépendant à apporter plus du fait que l'autre partenaire s'affranchit de ses besoins par un processus d'apprentissage et de captation des compétences qui lui faisaient défaut au début de la relation.

Une autre controverse concerne la répartition du capital. Certains auteurs pensent qu'elle a un effet direct sur les performances de l'alliance (Ramanantsoa, 1993). En ce sens, l'équilibre dans le partage du capital et le contrôle opérationnel, même s'il ralentit la prise de décision, évite les risques de «phagocytoses» abusives dans les relations asymétriques. D'autres auteurs soutiennent, par contre, qu'un équilibre est moins important dans le succès 
d'une alliance que l'importance des connaissances acquises (Hamel, Doz et Prahalad, 1989). Dans le cas des alliances internationales, Ohamé (1990) signale que la propension des entreprises étrangères à vouloir contrôler la majorité du capital est un «avatar de la culture capitaliste occidentale »: «[...] détenir $51 \%$ du capital vous donne juridiquement les pleins pouvoirs. Mais quel est ce pouvoir lorsqu'il s'agit du contrôle des activités sur un marché étranger dont vous ne savez pas grand-chose, éloigné comme vous l'êtes des besoins des clients, dans votre bureau à moquette rouge de Manhattan, de Paris ou de Francfort?» (Ohamé, 1990, p. 32).

De manière générale, de nombreux auteurs (Yan et Gray, 1994; Park et Ungson, 1997; Steensma et Lyles, 2000) ont insisté pour faire la distinction entre répartition du capital et contrôle parental de la relation, ce dernier étant défini par le degré d'influence d'un parent sur les décisions stratégiques et opérationnelles majeures de la relation coopérative (Yan et Zeng, 1999).

\subsection{Quelles options stratégiques pour les PME?}

Certains auteurs soutiennent que «dans les relations FMN-PME, ces dernières paraissent préoccupées par le court terme sans avoir une vision stratégique d'ensemble [...] Si les FMN ont des objectifs stratégiques, souvent les PME n'ont que des visions commerciales “amicales" » (O'Dwyer et O'Flynn, 2005, p. 415). D'autres auteurs font apparaître le caractère plus coopératif des PME et de leur personnel (Shan et Hamilton [1991] et Ronen et Shenkar [1985] cités dans Park et Ungson, 1997), à cela s'ajoutent les avantages «classiques » des PME en termes de flexibilité, de capacité d'adaptation et de rapidité de réaction. L'un des gros avantages des multinationales à taille mondiale est de pouvoir compter, du point de vue de l'exploitation, sur une structure administrative leur permettant d'organiser la production et l'approvisionnement sous la forme de réseaux (selon Porter, cité par Fernandez et Noel, 1993, p. 5).

Dans une publication relative aux alliances stratégiques comme réponse des PME à la mondialisation, Fernandez et Noel (1993) présentent une comparaison des actifs spécifiques des FMN, des PME mondiales et des PME locales. Ces auteurs montrent que les PME ne semblent pas trop défavorisées en termes d'innovation-produits, tandis que les FMN présentent un avantage en termes de R-D sur les procédés et dans le domaine des technologies. Les auteurs analysent trois types de réaction des PME: le maintien (sans croissance), le désengagement (retrait ou réorientation de l'activité) et, enfin, la croissance. En adaptant une grille à double entrée avec les options stratégiques, d'une part, et les positions de la PME par rapport à la FMN, d'autre part, ils aboutissent à six options. 


\section{TABLEAU 1}

Options stratégiques retenues pour une PME face à une FMN

\begin{tabular}{|lll|}
\hline Options & Interne & Externe \\
\hline Maintien & $\begin{array}{l}\text { Amélioration produit, } \\
\text { maîtrise des coûts. }\end{array}$ & Alliances stratégiques \\
Croissance & $\begin{array}{l}\text { Développement de nouveaux } \\
\text { marchés, augmentation de capacité. }\end{array}$ & $\begin{array}{l}\text { Alliances stratégiques } \\
\text { ou fusion }\end{array}$ \\
Désengagement & $\begin{array}{l}\text { Nouveaux couples produits-marchés } \\
\text { liquidation. }\end{array}$ & $\begin{array}{l}\text { Alliances stratégiques } \\
\text { ou cession }\end{array}$ \\
\hline
\end{tabular}

Source: Fernandez et Noel (1993), p. 12.

Les alliances occupent ainsi une place importante dans les figures stratégiques potentielles de la PME, quelle que soit sa position par rapport à une FMN (concurrent direct, fournisseur ou distributeur). Dans le cas d'une PME aux ressources limitées, les options stratégiques «internes» apparaissent compromises. Les auteurs indiquent que, dans le cas d'une concurrence frontale avec une FMN, «la seule option envisageable est un désengagement aussi rapide que possible pour pouvoir procéder, dans les conditions les plus avantageuses, à la vente de l'entreprise et dans le meilleur des cas à sa cession à l'entreprise mondiale peu après que celle-ci aura fait son entrée sur le marché» (Fernandez et Noel, 1993, p. 13).

Fernandez (1993) signale aussi les risques de telles relations pour les PME locales. D'une part, les rapports de forces apparaissent déséquilibrés au détriment des PME et, d'autre part, la FMN peut faire appel à la PME juste pour son installation sur le marché visé. Les scénarios envisagés varient selon les intentions stratégiques des deux partenaires, mais dépendent aussi de la manière dont est gérée la coopération. Cela va d'un rapprochement définitif (fusion/acquisition) à un transfert d'activité ou à une sortie d'alliance (par consensus ou rupture), en passant par des situations de rapprochement durable mais non irréversible et d'une autonomie de l'alliance par rapport aux deux partenaires.

\subsection{Alliances stratégiques et stade de développement du pays d'accueil}

De même que les références théoriques se rapportant aux relations entre FMN et PME sont rares par rapport à celles traitant des relations interFMN, les publications relatives aux alliances stratégiques dans des pays en 
développement ou en transition vers l'économie de marché demeurent rares en comparaison de celles traitant des alliances dans les pays en développement. Cependant, «les caractéristiques des joint ventures (JV) dans les PVD diffèrent de celles des JV dans les pays développés. Ces différences se situent en termes de stabilité de la relation, d'autonomie et de partage du capital, de motivations de création de la JV et du contrôle de la gestion » (Beamish et Banks, 1987, p. 9, traduction libre).

Quelques traits communs se rapportant au contexte du pays d'accueil ont été identifiés: la stabilité de la relation est plus sensible au contrôle de gestion dans un contexte de pays en développement (Park et Ungson,1997). Les attentes du partenaire local en termes d'apprentissage managérial sont plus grandes (les freins dans ce sens du partenaire étranger accentuant les risques de conflits) [Steensma et Lyles, 2000]. Les problèmes d'adaptation, de confiance et de dépendance (Gill et Butler, 2003), les problèmes de compatibilité de culture et de culture de management (Park et Ungson, 1997) apparaissent plus fréquents. L'attitude du pays d'accueil et son effet sur l'alliance sont accentués par le contexte de la relation (Franko, 1989; Fagre et Wells, 1982, cités dans Yan, 1998; Luo, Shenkar et Nyaw, 2001).

\subsection{Un cadre d'analyse des stratégies d'alliances entre PME et FMN}

Notre cadre d'analyse sera constitué de deux volets théoriques principaux:

- l'analyse stratégique: motivations et structure des deux partenaires et de la nouvelle entité (Garette et Dussauge, 1990), risque de dépendance et pouvoir de négociation (Inkpen et Beamish, 1997), pouvoir de marché et modification dans la configuration du secteur (Kogut, 1988, 1991);

- l'apprentissage organisationnel dans une optique stratégique:processus et domaines couverts (Ingham, 1994), freins, limites et protections des partenaires (Hamel, Doz et Prahalad, 1989) et processus dynamique d'apprentissage. 


\section{TABLEAU 2}

\section{Cadre théorique et questions de recherche}

\begin{tabular}{|c|c|c|c|c|}
\hline $\begin{array}{l}\text { Volet } \\
\text { théorique }\end{array}$ & Auteurs & $\begin{array}{l}\text { Objet de } \\
\text { l'analyse }\end{array}$ & Questions traitées & $\begin{array}{l}\text { Principaux } \\
\text { résultats }\end{array}$ \\
\hline $\begin{array}{l}\text { Analyse } \\
\text { stratégique } \\
\text { des alliances }\end{array}$ & $\begin{array}{l}\text { Garette et } \\
\text { Dussauge } \\
\text { (1990). }\end{array}$ & $\begin{array}{l}\text { Motivations } \\
\text { stratégiques } \\
\text { des partenaires. }\end{array}$ & $\begin{array}{l}\text { Sélection du } \\
\text { partenaire, } \\
\text { apports } \\
\text { respectifs Q1. }\end{array}$ & $\begin{array}{l}\text { Addition ressources } \\
\text { ou complémenta- } \\
\text { rités. }\end{array}$ \\
\hline $\begin{array}{l}\text { Théorie du } \\
\text { pouvoir de } \\
\text { négociation }\end{array}$ & $\begin{array}{l}\text { Inkpen et } \\
\text { Beamish } \\
(1997) \text {; } \\
\text { Yan et Gray } \\
\text { (1994). }\end{array}$ & $\begin{array}{l}\text { Déséquilibre } \\
\text { balance } \\
\text { du pouvoir } \\
\text { de négociation } \\
\text { et contrôle. }\end{array}$ & $\begin{array}{l}\text { Instabilité des } \\
\text { alliances asymé- } \\
\text { triques, processus } \\
\text { de négociation et } \\
\text { apprentissage } \mathbf{Q 2} \text {. }\end{array}$ & $\begin{array}{l}\text { Instabilité } \\
\text { provoquée par } \\
\text { l'apprentissage du } \\
\text { partenaire étranger. }\end{array}$ \\
\hline $\begin{array}{l}\text { Théorie du } \\
\text { pouvoir } \\
\text { de marché }\end{array}$ & $\begin{array}{l}\text { Harrigan } \\
\text { (1988); } \\
\text { Kogut (1988). }\end{array}$ & $\begin{array}{l}\text { Les alliances } \\
\text { stratégiques et } \\
\text { la concentration } \\
\text { du secteur. }\end{array}$ & $\begin{array}{l}\text { Effet «collusif» } \\
\text { des alliances stra- } \\
\text { tégiques } \mathbf{Q 3} \text { (c). }\end{array}$ & $\begin{array}{l}\text { Plus de } \\
\text { concentration et } \\
\text { motivation compé- } \\
\text { titive de l'alliance } \\
\text { stratégique. }\end{array}$ \\
\hline $\begin{array}{l}\text { Apprentissage } \\
\text { et transfert } \\
\text { organisationnels }\end{array}$ & $\begin{array}{l}\text { Ingham (1994); } \\
\text { Mowery, Oxley } \\
\text { et Silverman } \\
\text { (1996). }\end{array}$ & $\begin{array}{l}\text { Nature des } \\
\text { transferts } \\
\text { de savoir par } \\
\text { les alliances. }\end{array}$ & $\begin{array}{l}\text { Processus et } \\
\text { mécanismes des } \\
\text { transferts de } \\
\text { savoir } \mathbf{Q 3} \text { (a). }\end{array}$ & $\begin{array}{l}\text { Rôle important de } \\
\text { l'alliance comme } \\
\text { interface du } \\
\text { transfert. }\end{array}$ \\
\hline $\begin{array}{l}\text { Course/freins à } \\
\text { l'apprentissage }\end{array}$ & $\begin{array}{l}\text { Hamel, Doz } \\
\text { et Prahalad } \\
\text { (1989); Hamel } \\
\text { (1991). }\end{array}$ & $\begin{array}{l}\text { Course à } \\
\text { l'apprentissage } \\
\text { des partenaires. }\end{array}$ & $\begin{array}{l}\text { Mécanismes d'ap- } \\
\text { prentissage et de } \\
\text { freins. Protection } \\
\text { des compétences } \\
\text { Q3 (a). }\end{array}$ & $\begin{array}{l}\text { L'alliance comme } \\
\text { une «fenêtre»sur } \\
\text { les compétences } \\
\text { du partenaire. }\end{array}$ \\
\hline $\begin{array}{l}\text { AS } \\
\text { et asymétrie } \\
\text { et instabilité }\end{array}$ & $\begin{array}{l}\text { Parkhe (1991); } \\
\text { Steensma et } \\
\text { Lyles (2000); } \\
\text { O'Dwyer et } \\
\text { O'Flynn (2005). }\end{array}$ & $\begin{array}{l}\text { Rôle de l'asy- } \\
\text { métrie de taille } \\
\text { et de la diversité } \\
\text { entre parents sur } \\
\text { la stabilité de } \\
\text { l'alliance. }\end{array}$ & $\begin{array}{l}\text { Types, impor- } \\
\text { tance et effets } \\
\text { de la diversité } \\
\text { entre ressources } \\
\text { et cultures des } \\
\text { partenaires } \\
\text { Q1,2 et 3. }\end{array}$ & $\begin{array}{l}\text { Rôle positif des } \\
\text { complémentarités } \\
\text { en ressources et } \\
\text { négatif pour les } \\
\text { différences de } \\
\text { culture. }\end{array}$ \\
\hline AS PVD & $\begin{array}{l}\text { Beamish } \\
(1985) ; \\
\text { Sim et Yunus } \\
\text { (1998); Luo, } \\
\text { Shenkar et } \\
\text { Nyaw (2001). }\end{array}$ & $\begin{array}{l}\text { Comparaison des } \\
\text { alliances dans les } \\
\text { pays développés } \\
\text { et PVD. }\end{array}$ & $\begin{array}{l}\text { Analyse de la } \\
\text { spécificité des } \\
\text { alliances Nord- } \\
\text { Sud } \mathbf{Q 1 , 2} \text { et } \mathbf{3} \text {. }\end{array}$ & $\begin{array}{l}\text { Instabilité plus } \\
\text { importante } \\
\text { dans les PVD } \\
\text { et spécificité } \\
\text { des alliances } \\
\text { stratégiques. }\end{array}$ \\
\hline
\end{tabular}

Revue internationale P.M.E., vol. 21, n 1, 2008 


\subsubsection{L'analyse stratégique: motivations, pouvoir de marché et dépendance}

La première composante à analyser est l'adéquation entre les motivations et les apports croisés des deux partenaires. Que recherche chaque partenaire chez l'autre? (Garette et Dussauge, 1990.) La seconde question à traiter est d'établir les objectifs des deux partenaires à travers cet accord pour leur groupe (et leurs filiales). Autrement dit, comment insérer cet accord dans les stratégies globales des deux groupes?

Le second volet de l'analyse stratégique concerne les effets sur le secteur. L'analyse peut être faite dans les deux sens: structure du secteur/alliance et alliance/nouvelle structure du secteur (Burgers, Hill et Chan Kim,1993). Dans un article fondateur traitant des alliances stratégiques, Kogut (1988) analyse à travers la notion de pouvoir de marché la question des effets d'un nouvel accord sur la configuration d'une industrie. Cette analyse peut se résumer avec trois hypothèses:l'alliance est un investissement défensif pour l'un des partenaires contre les incertitudes de la concurrence; l'alliance est un moyen pour priver les autres concurrents d'un allié potentiel; l'alliance est un moyen (collusif) de stabiliser la concurrence et d'augmenter la concentration du secteur.

Hamel, Doz et Prahalad (1989) soutiennent que «l'alliance peut renforcer la position des deux sociétés vis-à-vis de leurs concurrents tout en fragilisant l'un des partenaires par rapport à l'autre». D'autres auteurs ne voient en cela qu'un transfert de la concurrence et non une modification profonde (Veron, 1998). Dans ce sens, l'alliance contribuerait à créer des «verrous stratégiques » pour que l'affrontement se fasse entre des coalitions et non pas entre entreprises isolées. La compétition est reportée à une échelle plus grande mais, en aucun cas, éliminée.

\subsubsection{L'apprentissage organisationnel: la compétition au sein de la coopération}

L'une des motivations essentielles du recours aux alliances stratégiques, notamment pour les PME, est l'accès aux compétences stratégiques du partenaire (la «fenêtre d dont parle Hamel,1991). L'approche par l'apprentissage constitue notre second volet théorique.

Entre deux «partenaires», l'apprentissage est influencé d'abord par l'existence de connaissances communes préliminaires à l'entrée dans la coopération (Ingham, 1994). Ensuite, d'autres déterminants sont à prendre en compte: l'intention d'internaliser les compétences du partenaire (ou la 
«volonté d'apprendre » pour Hamel, Doz et Prahalad, 1989), la transparence organisationnelle et la réceptivité du partenaire ou sa capacité d'absorption des nouvelles connaissances (Mowery, Oxley et Silverman, 1996).

L'apprentissage peut se faire sur trois plans (Ingham,1994): les savoirs et les savoir-faire techniques, la négociation du contrat et la gestion de l'accord et des conflits. Souvent, il donne lieu aussi à des préoccupations contradictoires (ou le dilemme du transfert de compétences; Larsson et al., 1998). Doit-on se comporter en bon partenaire pour accroître l'apprentissage collectif et l'émergence de connaissances inattendues (Ingham, 1994) ou protéger la diffusion de connaissances spécifiques et être opportuniste dans une course individuelle pour l'acquisition des compétences du partenaire pour se défaire de sa dépendance? (Hamel, Doz et Prahalad, 1989.)

Dans cette optique, l'alliance stratégique est une opportunité, pour les deux partenaires, de renforcer leurs compétences de base. Cependant, cet objectif se heurte à deux difficultés majeures: la nature des connaissances à transférer, souvent tacites et donc difficilement imitables et généralisables au niveau de l'organisation (Kogut, 1988), et la spécialisation des tâches, qui freine l'apprentissage des deux partenaires.

Concernant les relations d'alliances stratégiques asymétriques ${ }^{2}$, les notions de capacité d'absorption et de «digestibilité» sont souvent mises en avant pour expliquer certaines difficultés en termes d'apprentissage (Cohen et Levinthal, 1990; Hennart et Reddy, 1997). L'absence de compétences coopératives et la divergence des cultures managériales (Parkhe, 1991) peuvent constituer de sérieux freins à l'apprentissage organisationnel des partenaires, notamment pour la PME. L'asymétrie de taille et l'absence de relations antérieures entre les partenaires constituent selon certains les facteurs d'un apprentissage à sens unique, en faveur du partenaire dominant (Inkpen et Beamish, 1997; Park et Ungson, 1996).

\section{2. Étude de cas Danone - Djurdjura en Algérie}

Le cas Danone-Djurjura permet de traiter une relation asymétrique (Danone, FMN et Djurjura, PME), dans un contexte de pays en transition (Algérie). Nous exposerons dans cette partie empirique, la méthode retenue puis les résultats obtenus.

2. Un numéro spécial (vol. 10, $\mathrm{n}^{\circ}$ 1, automne 2005) de la revue Management international (HEC Montréal) a été consacré à ce thème. 


\subsection{Contexte empirique et méthodologie}

Danone, leader mondial des produits laitiers frais, constitue un bon exemple de «firme globale». Djurdjura, PME algérienne spécialisée dans le secteur des produits laitiers frais (PLF) est typique de l'essor du capitalisme privé dans un PVD. Le rapprochement entre les deux entreprises a conduit à la création de Danone-Djurdjura Algérie (DDA), contrôlée à $51 \%$ par Danone depuis 2001 . Les $49 \%$ restants sont détenus par le groupe Batouche.

TABLEAU 3

\section{Danone et Djurdjura en 2004}

\begin{tabular}{lll}
\hline & Danone (le groupe) & Djurdjura \\
\hline C.A. & $\begin{array}{l}\text { 13 } 700 \text { millions d'euros } \\
31 \% \text { des ventes réalisées dans les pays émergents }\end{array}$ & 52 millions d'euros \\
Effectif & $\begin{array}{l}89449 \text { personnes } \\
\text { (dont } 74 \% \text { en dehors de l'Europe occidentale) }\end{array}$ & 631 personnes \\
Position & $\begin{array}{l}\mathrm{N}^{\circ} 1 \text { mondial pour les PLF et les eaux minérales } \\
\text { No } 2 \text { pour les biscuits et produits céréaliers }\end{array}$ & $\begin{array}{l}\text { No 1 en Algérie } \\
\text { pour les PLF }\end{array}$ \\
\hline
\end{tabular}
majeures:

L'Algérie est un pays en transition du fait de plusieurs évolutions

- le code d'investissement adopté en 1993 favorise l'implantation de firmes multinationales avec plusieurs dispositions (rapatriement des bénéfices, législation du travail, etc.);

- à partir de la fin de 1998 (date de la fin du programme d'ajustement structurel), certaines multinationales se sont installées en Algérie, notamment dans le secteur agroalimentaire;

- le secteur privé en Algérie connaît une dynamique forte depuis les années 1990; le secteur agroalimentaire ne compte pas moins de 150000 PME, concentrées dans les grands pôles industriels du pays (Blida, Alger, Bejaia, Oran, etc.).

L'étude longitudinale du cas Danone - Djurdjura est justifiée d'abord par la complexité des questions abordées (asymétrie, contexte de pays en développement), ensuite, par la nature dynamique de la problématique (processus de négociation et d'apprentissage, changement organisationnel) et, enfin, par l'accès aux informations (nécessité de mener des entretiens approfondis). 


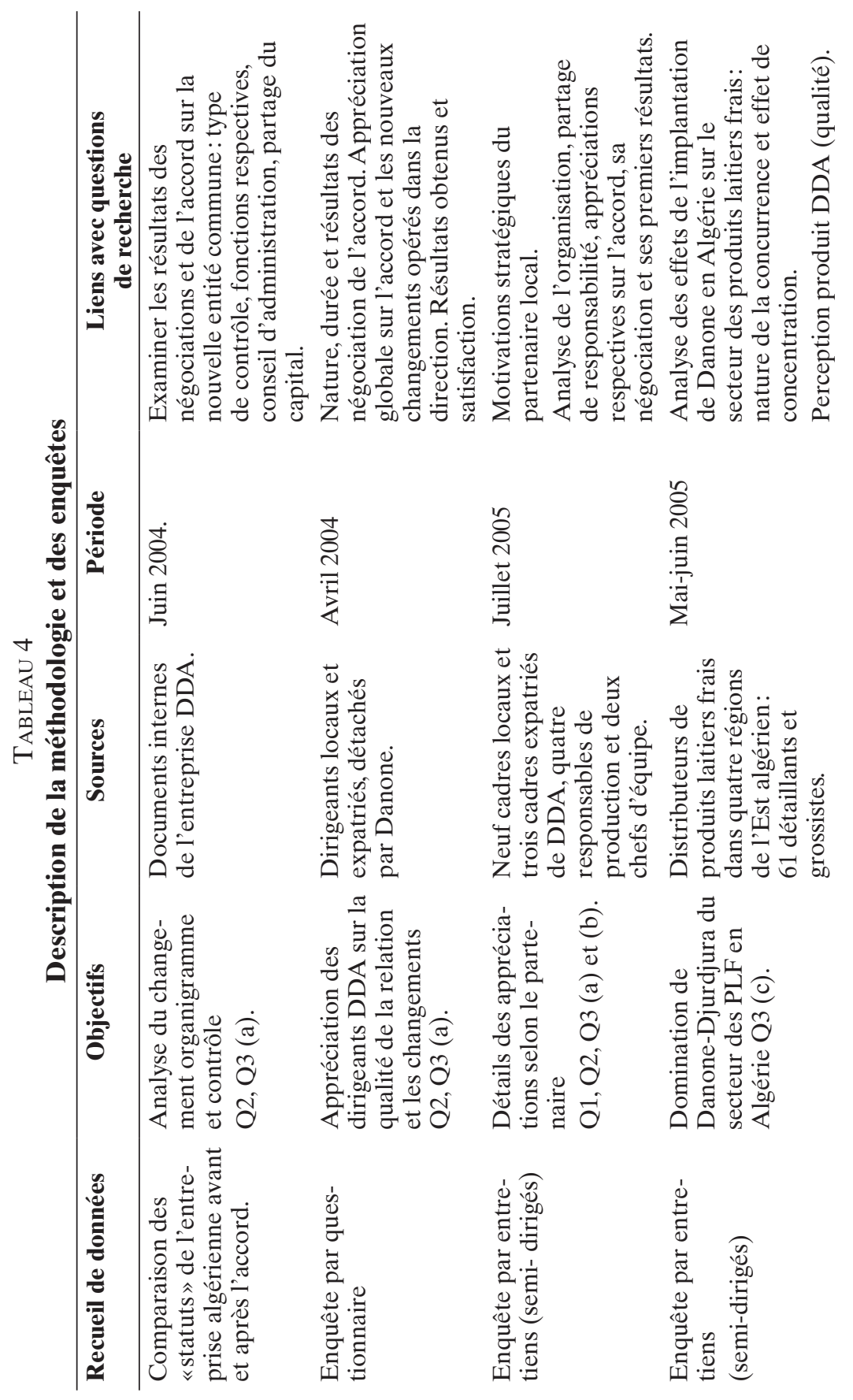

Revue internationale P.M.E., vol. 21, nº 1, 2008 
Nous avons mené deux types d'enquêtes. La première, destinée aux dirigeants de la PME, avait pour but de cerner les motivations de l'entreprise et de comprendre le déroulement des négociations. Elle comprenait deux volets: un questionnaire préliminaire en trois blocs de questions, chaque bloc étant relatif à l'une de nos trois questions de recherche (motivations, déroulements et résultats) et une série d'entretiens menés durant le mois de mai 2005 auprès de cadres de la PME et de responsables de la production et de la qualité. Ces entretiens semi-directifs ont été menés en face à face auprès de 18 membres du personnel de DDA dont 12 cadres supérieurs ( 3 cadres étrangers), 4 responsables de production et 2 chefs d'équipes. Ils ont duré en moyenne une heure et demie et ont permis le recueil d'informations en compléments des renseignements recueillis à travers le questionnaire.

La seconde enquête visait les distributeurs. Elle a concerné 61 détaillants et grossistes de produits laitiers frais à travers quatre wilayas (régions) de l'Est algérien. Cette enquête a permis d'apprécier les effets de l'implantation de Danone à travers DDA sur la configuration du secteur des produits laitiers frais (PLF) et les changements dans la structure compétitive. Les questions portaient sur l'appréciation de la qualité du produit et de son emballage, la largeur de la gamme et les volumes vendus.

Nous avons enfin eu recours à des documents internes de l'entreprise DDA et du groupe Batouche ou à des sources secondaires d'information (coupures de presse, rapports et monographies) afin de compléter notre analyse des effets de l'accord.

\subsection{Les modalités de l'accord Danone-Djurdjura}

\subsubsection{Déroulement des négociations et évaluation des actifs}

Les premiers contacts ont été effectués par Danone auprès de la PME algérienne à la fin de l'année 1999 en vue de la signature d'un accord de partenariat entre le groupe Danone et le groupe familial Batouche, propriétaire de Djurdjura. Les négociations ont duré deux ans et se sont déroulées pour l'essentiel en France. L'entreprise Djurdjura avait délégué des cadres et pris contact avec des experts financiers algériens. La délégation était conduite par le directeur général et principal actionnaire de l'entreprise ${ }^{3}$.

Les problèmes d'évaluation des actifs, notamment du côté de Djurdjura, ont conduit les deux partenaires à opter pour la désignation d'un commissaire aux apports par un tribunal algérien pour l'estimation de la situation

3. Résultats de l'enquête par questionnaire auprès des cadres dirigeants de DDA, avril 2005. 
financière de l'entreprise afin de procéder à une augmentation de son capital. Le groupe Danone a eu recours à un bureau de conseil financier, le cabinet SWICORP, pour l'accompagner dans ses négociations avec le groupe Batouche. Après la réévaluation du capital de l'entreprise, Danone s'est engagée à racheter 51 \% des parts, soit 1377000 actions de 1000 dinars chacune (environ 10 euros).

L'accord, qui a donné lieu à la création de la nouvelle entité par le dépôt des nouveaux statuts de l'entreprise auprès d'un notaire agréé en Algérie, a porté à la fois sur les engagements réciproques des deux partenaires et sur les modalités de rupture en cas de désaccord. Cet accord s'apparente à une véritable alliance stratégique selon les caractéristiques de la définition retenue. Cette alliance peut être considérée comme stratégique pour la PME algérienne et accessoire pour le groupe Danone car ne concernant que le marché algérien et le seul métier des yaourts et produits laitiers frais et donc de faibles enjeux en termes de chiffre d'affaires, au moins à moyen terme.

\subsubsection{Le contrôle de l'alliance}

Il existe entre les deux partenaires de l'alliance étudiée une forte asymétrie de taille. L'organigramme de la direction de DDA confirme l'hypothèse d'un contrôle accru de DDA par le groupe Danone. En effet, hormis la direction technique de l'usine et la direction des ressources humaines, toutes les autres directions ont à leur tête un cadre du groupe Danone.

\section{FiguRE 1}

\section{Organigramme de la direction de DDA}

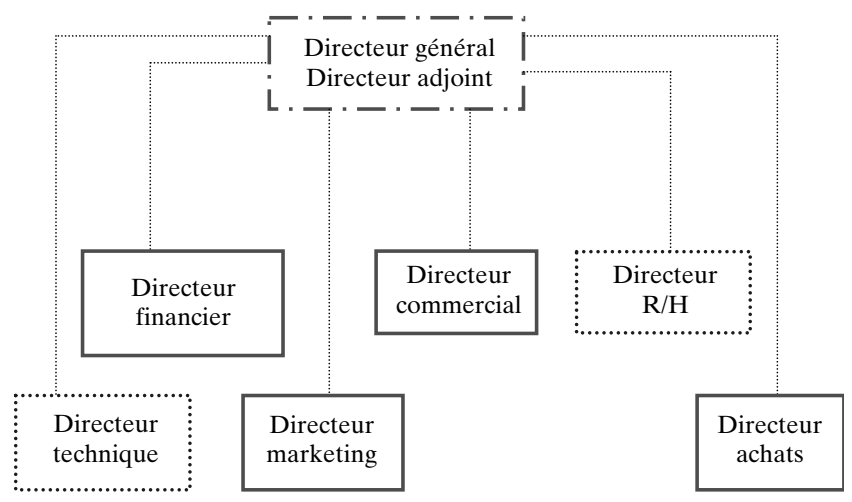

Note: Les fonctions signalées en lignes continues sont assurées par des cadres du groupe Danone. Celles en pointillés sont occupées par les anciens cadres algériens de l'entreprise.

Source: Entretiens avec le directeur des ressources humaines de DDA, 2004. 
À première vue, la PME n'a aucune marge de manœuvre stratégique. Toutefois, les entretiens effectués auprès des cadres de DDA, détachés par Danone, et l'examen des statuts de DDA mettent en évidence le fait que l'entreprise Djurdjura est relativement associée à la prise de décision. Par exemple, le directeur financier et comptable de DDA, cadre détaché du groupe Danone, affirme que:

J'ai déjà effectué une rotation comme cadre du groupe pour nos implantations dans les pays de l'Est. J'ai travaillé en République tchèque, en Roumanie et en Bulgarie. Mais avec DDA, nous avons une situation inédite pour le groupe Danone. Nous contrôlons 51 \% du capital mais pas le conseil d'administration. Les décisions se prennent à $80 \%$ (4/5) des voix et nous n'en avons que la moitié (au prorata du nombre d'actions détenues). Nous devons de ce fait obtenir l'accord de notre partenaire pour toutes les décisions importantes, y compris les plans stratégiques à moyen terme et les décisions d'investissement.

Le cadre de Danone parle de situation «inédite» ${ }^{4}$. Danone, qui détient $51 \%$ du capital, doit cependant consulter son partenaire pour toutes les décisions stratégiques. Les statuts de l'entreprise stipulent que le conseil d'administration qui compte 13 membres ( 7 de Danone et 6 de Djurdjura) « délibère à la majorité qualifiée (4/5 des membres) pour les décisions suivantes ${ }^{5}$ :

- nomination ou révocation du directeur général et du directeur général adjoint et fixation de leur rémunération;

- approbation ou toute révision du plan, du plan révisé et du budget annuel;

- acquisition ou création d'une nouvelle activité ou d'une nouvelle société d'un montant excédant 100000 dinars $^{6}$;

- toute cession d'une partie importante des activités représentant plus de $10 \%$ du chiffre d'affaires de la société ou du patrimoine de la société;

- toute opération de cession et/ou d'investissement dont le montant est supérieur de $10 \%$ ou plus au montant prévu dans le plan, le plan révisé ou le budget annuel;

4. En fait, elle ne l'est pas pour le groupe. Plusieurs autres cas font état de situation où Danone contrôle la majorité du capital sans avoir la majorité dans le conseil d'administration.

5. Extrait des statuts de l'entreprise Danone-Djurdjura Algérie; Article 19: délibérations du conseil d'administration.

6. Soit approximativement 1000 euros.

Revue internationale P.M.E., vol. 21, nº 1, 2008 
- l'établissement de la politique de sécurité alimentaire, notamment en matière d'organismes génétiquement modifiés.

Ainsi, les cadres de Djurdjura sont associés à la prise de décision et peuvent bloquer toute tentative de prise de contrôle total de Danone.

\subsubsection{Motivations stratégiques des partenaires}

Les motivations des deux partenaires sont différentes. Pour la PME, l'accord est d'abord motivé par une stratégie défensive face à un concurrent potentiel et, ensuite, par les possibilités de bénéficier de la notoriété et de l'image de marque de la multinationale. Ainsi, les cadres interrogés donnent dans un ordre décroissant de priorité les motivations suivantes de l'accord $\mathrm{DDA}^{7}$ : notoriété et image de marque Danone, protection face à la concurrence étrangère et locale (dont celle du groupe Danone s'il venait à s'installer sur le marché algérien, seul ou avec un autre partenaire), apport en techniques de gestion et procédures de qualité.

Notre enquête montre que ni les difficultés financières et l'apport en capitaux et liquidités, ni même le transfert des techniques de production ne semblent constituer des motivations suffisantes pour la signature de l'accord (notre hypothèse 1 est donc rejetée). Cela est notamment confirmé par les entretiens menés auprès des cadres de DDA: «[...] concernant la vision stratégique de Djurdjura, l'accord avec Danone apparaît comme une opportunité pour grandir, accroître notre part de marché et avoir une chance de nous insérer dans une économie mondiale. Nous n'avions pas le choix, c'était faire cela ou disparaître. Il nous faut dépasser les visions impérialistes d'antan. Danone est un partenaire "de taille", et nous comptons profiter de son image de marque et de sa notoriété ${ }^{8}$.

Un autre cadre de DDA explique, pour sa part, que «Danone a contacté Djurdjura et conclu cet accord avec nous parce que nous étions et nous sommes encore leader sur notre marché. Nous avons un réseau de distribution assez efficace et une part de marché non négligeable. Les apports de Danone peuvent s'identifier à travers l'amélioration de la gestion de l'entreprise et la mise en place des procédures pour le contrôle et l'amélioration de la qualité. Nous ne pouvons plus concevoir l'avenir de DDA sans Danone ${ }^{9}{ }$.

7. Ces trois raisons sont les plus fréquemment citées sur une liste de neuf (dont les difficultés financières).

8. Entretien avec l'ex-DRH, mai 2005 (négociateur et conseiller du DG pour l'accord avec Danone).

9. Entretien avec l'ex-DRH de DDA, membre de la délégation de négociation, mai 2005. 
De son côté, Danone semble privilégier les connaissances du partenaire local concernant les habitudes de consommation et du marché local, d'une part, le réseau de distribution et de sous-traitants de Djurdjura, d'autre part. On pourrait penser, dans une perspective d'analyse stratégique (Kogut, 1988; Garette, 1989), que Danone vise aussi à s'accaparer d'une position de marché déjà existante (celle du leader en l'occurrence) et contrôler ainsi un concurrent potentiel. «Djurdjura nous apporte ses connaissances du climat algérien des affaires. Aussi, nous bénéficions d'un réseau très performant de sous-traitants et de distributeurs que nous comptons bien évidemment développer ${ }^{10}$.»

\subsection{Les effets de l'accord Danone Djurdjura Algérie (DDA)}

Ces effets peuvent s'évaluer en termes économiques, techniques et organisationnels.

\subsubsection{Effet de l'accord sur Danone Djurdjura Algérie}

Avec les efforts faits en termes d'investissement, les résultats financiers ne tardèrent pas à suivre. En 2004, le chiffre d'affaires a augmenté de 87 \% par rapport à 2001. Les ventes ont connu une augmentation de $30 \%$ alors que l'effectif n'a évolué que de $12 \%$.

TABLEAU 5

Résultats de Djurdjura de 2001 à 2004

\begin{tabular}{lrrrrc}
\hline & $\mathbf{2 0 0 1}$ & $\mathbf{2 0 0 2}$ & $\mathbf{2 0 0 3}$ & $\mathbf{2 0 0 4}$ & Variation 2001-2004 \\
\hline Effectif & 541 & 559 & 590 & 631 & $\mathbf{1 2 \%}$ \\
\hline Frais du personnel* & $/$ & 160 & 201 & 306 & $/$ \\
\hline Chiffre d'affaires* & 2800 & 2424 & 3980 & 5222 & $\mathbf{8 7 \%}$ \\
\hline $\begin{array}{l}\text { Volumes } \\
\text { des ventes** }\end{array}$ & 59297 & 44049 & 64883 & 77403 & $\mathbf{3 0 \%}$ \\
\hline
\end{tabular}

* En millions de dinars (1 euro = 95 dinars $)$, C.A. de 2001, estimation.

** En tonnes.

Source: DDA, département des finances et de la comptabilité.

L'examen des chiffres présentés dans le tableau ci-dessus révèle une forte augmentation du chiffre d'affaires par rapport à celle de la production. Cela traduit une meilleure valorisation des produits Danone par l'augmentation des prix et le lancement de produits plus coûteux (Danao, Dan'up, Fruix, Yaourt brassé aux fruits, etc.).

10. Entretien avec le directeur financier et comptable (cadre du groupe Danone), mai 2005. 
En 2002, l'entreprise enregistre un recul de son chiffre d'affaires et de sa production. Cet état de fait peut être dû à la situation sociopolitique particulière de la région à cette époque. Une seconde «explication» moins intuitive nous a été communiquée par un responsable de DDA:

Avant la venue du groupe Danone, Djurdjura, comme la quasi-totalité des entreprises algériennes, sous-facturait ses ventes à la demande des clients. Lorsque ce dernier commandait, par exemple, 500 palettes de crèmes «dessert», il demandait à ce que sa facture ne contienne que 300 palettes. L'écart non facturé de 200 palettes n'était pas déclaré et permettait d'«économiser» sur les impôts à payer. Avec la venue de Danone, cette pratique n'était plus possible car la firme exigeait par déontologie que toutes les ventes soient facturées. Le même client qui commandait 500 palettes dont 200 non facturées n'en demandait alors plus que 300. Plusieurs clients se sont détournés de DDA pour s'approvisionner chez des entreprises concurrentes ${ }^{11}$.

Ce changement dans les pratiques commerciales de l'entreprise, pourtant courantes chez les concurrents, au profit d'un respect de la législation, est animé par un souci constant de la préservation de l'image de marque de Danone et de contrôle efficace des recettes commerciales.

En résumé, l'arrivée de Danone s'est accompagnée d'une amélioration des capacités de fabrication, des conditions de production et de la qualité des produits. On a également constaté une progression des résultats financiers et un changement des pratiques comptables et commerciales, dans le sens d'une plus grande transparence. Cependant, la nouvelle entité DDA est de plus en plus confrontée à de nouveaux problèmes: harmonisation des salaires et revendication des travailleurs, procédures de mise en conformité de la qualité et de l'hygiène, pressions sur les travailleurs pour augmenter la productivité, etc.

\subsubsection{De nouveaux enjeux pour une nouvelle organisation}

Dès l'entrée en vigueur de l'accord avec Djurdjura, Danone avait explicitement opté pour trois axes de développement: la modernisation de l'outil de production par de nouveaux investissements, la mise en place d'une politique de gestion des ressources humaines et, enfin, l'élaboration d'un programme dans le domaine de la sécurité alimentaire.

Concernant les ressources humaines, Danone s'est vite trouvée confrontée à une situation difficile: absence de politique de recrutement, rapports sociaux particuliers entre travailleurs, encadrement insuffisant, etc. Deux actions prioritaires ont été menées après l'arrivée de Danone pour la

11. Extrait de l'entretien avec un responsable du département AQSA, mai 2005.

Revue internationale P.M.E., vol. 21, nº 1, 2008 
réorganisation de la direction des ressources humaines: une harmonisation et une revalorisation substantielle des salaires avec le recrutement de nombreux cadres d'entreprises concurrentes et le lancement de programmes de formation, notamment pour le personnel technique. Les responsables techniques de DDA ont ainsi bénéficié d'une mise à niveau après l'installation de la ligne de production DANAO. Deux stages ont été effectués au Maroc et en Suède et des formations sur le site ont été organisées par des cadres du groupe Danone. Le personnel du département qualité et sécurité alimentaire bénéficie actuellement de stages à l'étranger dans le cadre de la mise en place du système HACCP et en vue de la certification aux normes ISO.

Concernant les conditions de travail, selon les résultats des entretiens effectués avec les chefs d'équipe de production et quelques travailleurs, il semblerait que les conditions d'hygiène se soient nettement améliorées. Par contre, de fortes pressions sont ressenties par les travailleurs en vue d'améliorer de manière continue les rendements et les procédures de travail. Les changements survenus dans le domaine du marketing ont eu pour effet d'accroître encore la pression sur les équipes de production. Une dizaine d'équipes travaille en triple rotation de huit heures pour assurer la continuité de la production des différentes lignes, sept jours sur sept.

Danone a mené, dès son installation, un programme rigoureux pour l'amélioration des conditions d'hygiène et la réduction des défauts de fabrication dans le cadre des «bonnes pratiques» de fabrication et d'hygiène (BPH et BPF) et de la «charte qualité de Danone» appliquée dans tous les sites de fabrication à travers le monde.

Deux faits témoignent de ces nouvelles orientations: la mise en quarantaine systématique des produits pendant 24 heures avant leur «libération» et la destruction automatique de tous les produits comportant un défaut de fabrication, même minime.

L'audit initié par AIB consulting, avec la participation des cadres techniques du département «assurance de la qualité et sécurité alimentaire», a permis une amélioration continue des conditions d'hygiène: à titre d'exemple, toutes les machines des différentes lignes de production sont arrêtées et nettoyées (stérilisées) toutes les deux heures.

En ce qui concerne l'innovation produit et procédés, DDA semble avoir délégué ses pouvoirs au groupe Danone. En effet, tous les lancements de nouveaux produits obéissent à la même démarche: briefing de la direction marketing (dirigée par une équipe de cadres Danone et détachée de l'unité de production), analyse des données et sondages, contact avec une unité du groupe Danone de fabrication des nouveaux arômes (basée, pour l'Afrique et le Moyen Orient, à Barcelone). Souvent, les produits lancés sur le marché 
algérien sont transposés à d'autres pays (notamment en Europe) : Petit suisse Gervais, Fruix, Danao, Dan'up, etc. Par souci de rationalisation des coûts, le nombre de références a été réduit de 72 en 2001 à 18 en $2004^{12}$, alors que les entreprises concurrentes continuent de lancer de nouveaux produits et innovent en termes d'arômes; Soummam a ainsi lancé les yaourts au miel et aux céréales en 2004.

\subsubsection{Les effets de l'accord sur le groupe «Batouche»: des synergies retrouvées}

Le groupe Batouche est constitué de cinq filiales autonomes spécialisées dans l'emballage papier et plastique et les industries agroalimentaires: produits laitiers et dérivés, viandes et transport frigorifique, plastique, cartons et papiers, yaourt et épicerie sèche. L'accord Danone Djurdjura Algérie a eu des effets sur ces différentes filiales.

FiguRE 2

\section{Composition du groupe algérien Batouche}

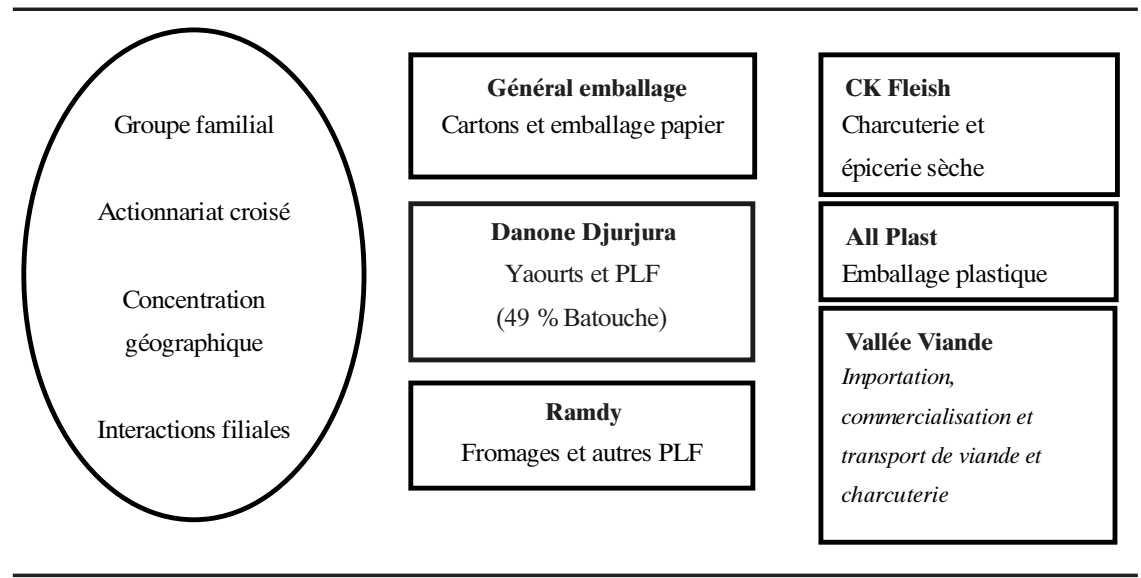

Source: Établie à partir des entretiens avec les dirigeants de DDA.

Bien avant l'accord DDA, la laiterie Djurdjura entretenait d'intenses relations avec les autres filiales du groupe. En effet, le groupe avait envisagé, dès les années 1990, une intégration verticale et internalisé des activités d'emballage et de transport frigorifique. Trois entreprises sont alors créées. All Plast pour l'emballage plastique, Général emballage ( $\mathrm{n}^{\circ} 2$ sur le marché algérien) pour l'emballage papier et carton et, enfin, Vallée viande, pour

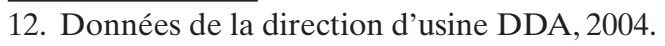

Revue internationale P.M.E., vol. 21, nº 1, 2008 
l'importation, le transport et la commercialisation de viandes. Les activités yaourt et fromages sont séparées. La filiale Ramdy produit et commercialise sous la marque Djurdjura mais aussi sous la marque Ramdy les produits laitiers frais. La laiterie Djurdjura se spécialise dans la production et la commercialisation de yaourts et de desserts lactés. Une dernière filiale du groupe est créée pour l'épicerie sèche et la charcuterie: CK Fleish.

Le groupe a également créé un réseau très important de sous-traitants et de distributeurs dont l'activité concerne tous les produits du groupe. Des synergies ont été développées entre les différentes filiales. La structure de gouvernance est caractérisée par un contrôle et un management familial: les cinq frères Batouche se trouvent à la tête des différentes filiales. Tous détiennent des actions dans les autres filiales de sorte qu'ils siègent dans les cinq conseils d'administration.

Cette configuration conglomérale, fréquente dans les pays méditerranéens (Rastoin, Ghersi et Tozanli, 2003), associée à une forte proximité géographique, permet aux différentes entreprises du groupe de réaliser des économies d'échelle importantes. Avec l'arrivée de Danone, ces synergies ont été renforcées. Ainsi, l'entreprise DDA sous-traite aux filiales du groupe ses activités de transport, de logistique et d'emballage. Il est aussi envisagé de créer une entreprise de collecte de lait cru pour la fourniture de l'atelier Danao. Cependant, les liens de plus en plus intenses, entretenus par DDA avec les autres filiales, risquent de créer des situations de dépendance et de perte de contrôle du groupe sur ses différentes activités.

Pour illustrer les nouvelles synergies entre les filiales du groupe Batouche stimulées par l'accord passé avec Danone, nous présenterons l'exemple du produit Dan'up. Il faut signaler que la quasi-totalité des activités du groupe est centrée autour de DDA. L'expansion de celle-ci a entraîné la croissance du chiffre d'affaires de trois des quatre autres filiales (à l'exception de CK Fleish).

Dan'up est l'un des produits lancés par DDA après l'arrivée de Danone. L'atelier de production a nécessité la construction d'une nouvelle plate-forme et l'acquisition d'une nouvelle ligne de production de 8500 bouteilles par heure. Quatre des cinq filiales du groupe participent dans la production du produit fini. Le remplissage des bouteilles de Dan'up s'effectue dans l'usine DDA. 


\section{Figure 3}

\section{Interactions entre les filiales du groupe Batouche: l'exemple de «Dan'up »}

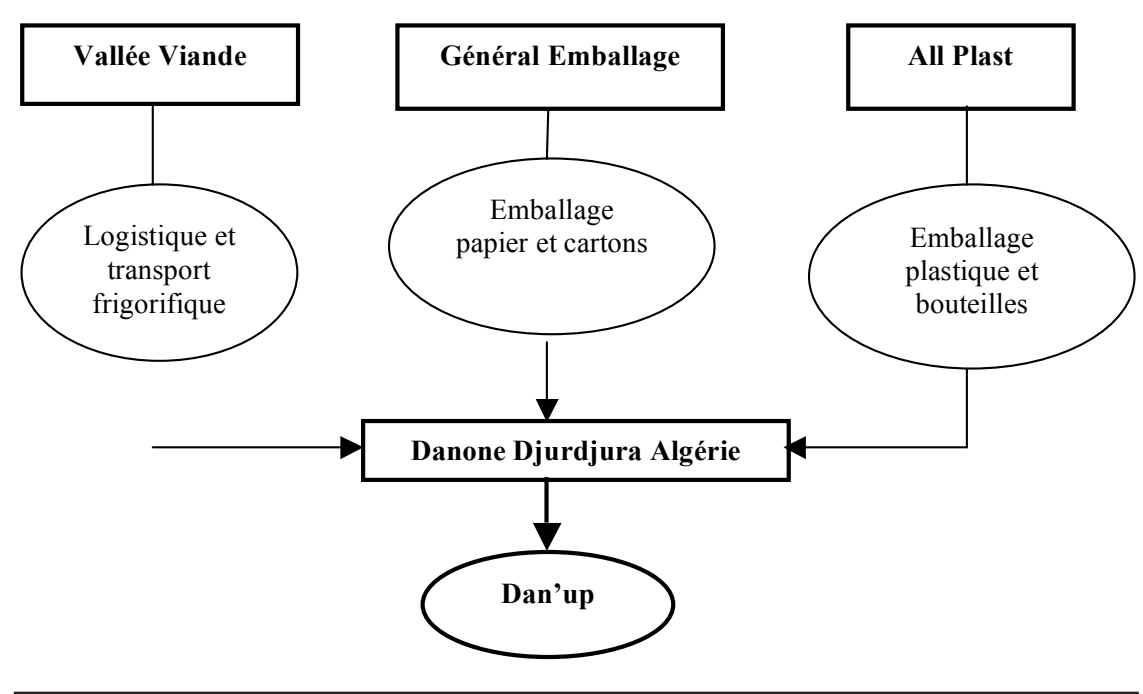

Source: Élaborée à partir des entretiens DDA et la visite de l'usine, avril-mai 2005.

Le choix des filiales du groupe Batouche obéit d'abord à une logique financière de minimisation des coûts. En effet, à cause de leur proximité géographique et de leurs relations privilégiées avec DDA, ces filiales constituent les premiers sous-traitants de l'entreprise. De même, DDA est le premier client de All Plast et de Général Emballage. DDA constitue aussi le meilleur débouché pour Vallée Viande à travers ses activités de transport frigorifique. Cette entreprise dispose de moyens importants (semi-remorques frigorifiques et relais de chambres froides) et devient essentielle pour les opérations logistiques de DDA.

\subsubsection{Les effets de l'accord sur le secteur des produits laitiers frais}

Avant l'arrivée de Danone en Algérie en 2001, le marché des yaourts comptait deux entreprises leaders (Djurdjura et Soummam) implantées toutes deux dans la wilaya de Béjaia à l'est du pays et une petite entreprise en forte croissance (Trèfle) localisée au centre. Les filiales du groupe étatique Giplait commençaient alors leur recentrage et abandonnaient progressivement la production et la commercialisation de yaourts. 
L'ouverture du marché algérien date du début des années 1990, mais des facteurs politiques et administratifs ont retardé l'arrivée des entreprises étrangères. À partir de 1998, un nombre important d'opérations de partenariat a été enregistré dans les secteurs de la pharmacie, de la chimie, de la mécanique, des banques et des assurances. En 2001, l'installation de Danone en partenariat avec le leader national de l'époque marqua le début d'une restructuration du marché des yaourts en Algérie.

Les résultats commerciaux obtenus par DDA sont en forte progression depuis l'arrivée de Danone ( $c f$. tableau 5). Selon, un cadre dirigeant de l'entreprise, la part de marché est passée de $25 \%$ à $35 \%$ entre 2001 et 2003.

\subsubsection{Une nouvelle configuration du marché}

Tout en permettant un renforcement des positions de marché des partenaires, les alliances stratégiques exercent une influence sur la structure du marché concerné. Dans le cas du marché des produits laitiers frais en Algérie, l'implantation de Danone a été concomitante à deux mouvements qu'il convient de décrire avant de présenter les évolutions du marché13:

- Des efforts considérables en termes de marketing et d'action commerciale ont été réalisés par DDA avec l'utilisation de tous les supports publicitaires et particulièrement les médias de masse. En parallèle, des tombolas, des jeux et des campagnes de sponsoring accompagnaient les lancements de nouveaux produits. DDA consacrait en 2003,3 \% de son chiffre d'affaires à des actions marketing.

- Ces efforts soutenus, conjugués à des facteurs externes (pouvoir d'achat, déterminants de la consommation et accès aux produits), ont abouti à l'élargissement de la demande globale de PLF en Algérie ${ }^{14}$. Ainsi, DDA estime avoir participé à hauteur de $40 \%$ à l'augmentation de la demande de PLF entre 2001 et $2003^{15}$.

Cet élargissement de la demande globale rend le marché algérien porteur et, de ce fait, conduit à l'accroissement du nombre d'intervenants. Cinq mouvements ont été ainsi observés :

13. Les mêmes évolutions ont été observées de manière plus prononcée dans le secteur des boissons non alcoolisées et sodas avec l'arrivée des deux géants Pepsi et Coca Cola.

14. La demande augmente ainsi pour les produits de Danone, à la suite de son effort marketing mais aussi pour les produits des marques concurrentes.

15. Interview de Claude Jolly, op. cit. 
- L'arrivée d'autres marques mondiales: Yoplait (Groupe Sodiaal) s'installe en Algérie avec une licence accordée à un partenaire algérien en 2003; la concurrence avec Danone pourrait se faire dans ce cas par la marque et la différenciation des produits.

- Le renforcement du pouvoir de marché des entreprises nationales existantes (Trèfle et Soummam) avec des efforts soutenus en termes d'innovation et un nombre important de nouveaux produits lancés.

- L'émergence de petits producteurs locaux pour les produits banalisés et appliquant une stratégie de concurrence par les prix; Hodna est créée en 2002 et s'installe à Msila dans le Sud-Est algérien; Palma Nova est implantée à Constantine et débute son activité en 2004; les deux entreprises produisent essentiellement des yaourts aromatisés.

- La reconversion d'autres producteurs: Candia (Groupe Sodiaal), installée en Algérie depuis 2000 par un accord de franchise avec une entreprise locale Tchin Lait, lance en 2004 un yaourt aux fruits à boire, concurrent direct du Danao de Danone.

- L'arrivée de concurrents potentiels mais sur des marchés différents : en avril 2005, Nestlé signe un accord de partenariat avec une entreprise locale (Sidi Kébir) pour la production et la commercialisation d'eau minérale et de lait en poudre.

On notera donc le nombre croissant des concurrents, leur forte concentration géographique mais aussi la diversité des modes d'implantation:Yoplait (sous licence), Candia (accord de franchise), et partenariat avec des entreprises locales pour Danone ou encore Nestlé. Pour défendre sa position de leader, Danone se trouve confrontée à des entreprises appliquant des stratégies différentes: affrontement par les prix pour les petits producteurs (Hodna et Palma Nova) et sur des produits d'entrée de gamme, concurrence par la marque et la différenciation pour les producteurs de taille importante (Soummam, Trèfle) ou détenteurs de marques mondiales (Yoplait) et hyper-segmentation pour les entreprises visant un produit spécifique (Candia pour le yaourt à boire).

\subsubsection{Les contraintes futures du marché des PLF}

Les efforts soutenus de DDA en termes de marketing conduisent à un élargissement de la demande de produits laitiers frais. Une nouvelle configuration du marché se construit avec l'arrivée de nouvelles entreprises étrangères, l'émergence de petits producteurs locaux et la reconversion de certaines entreprises alimentaires. 
L'enquête menée auprès des dépositaires et détaillants de quatre wilayas de l'Est algérien a permis de montrer que les entreprises pouvaient être classées en trois catégories distinctes: Danone et Soummam, comme leaders nationaux, Trèfle et Yoplait, comme concurrents directs et Hodna et Palma Nova, comme petits producteurs géographiquement localisés. L'enquête a aussi révélé que la concurrence entre les producteurs se faisait davantage en termes de délais de livraisons et de gamme de produits. La qualité du produit et de l'emballage semble être maîtrisée par les différents intervenants et ne constitue donc plus un facteur concurrentiel. La nouvelle configuration apparaît ainsi classique. On s'achemine vraisemblablement vers un oligopole à franges localisées (Rastoin, 1994). La concurrence se fera alors par une différenciation entre les quatre entreprises de tête et par les coûts pour les petits producteurs. En ce qui concerne le business model (différenciation), les efforts d'innovation entrepris par les leaders nationaux (Trèfle et Soummam) leur assurent une certaine avance sur les entreprises étrangères qui se limitent, pour l'instant, à réintroduire des produits déjà lancés (ou en phase de déclin) dans leurs pays respectifs.

Dans les années à venir, trois inconnues devraient être prises en compte dans l'élaboration des stratégies des entreprises de produits laitiers frais. D'abord, le ralentissement probable de la demande alimentaire à moyen terme. Ainsi, le coefficient budgétaire des dépenses alimentaires, qui était de $52 \%$ en 1990 (Rastoin et Tozanli, 1992), a baissé à $45 \%$ dans les années $2000^{16}$. Même si elle demeure forte en termes absolus, la demande de produits alimentaires connaîtra des évolutions identiques à celles observées au nord de la Méditerranéenne: ralentissement et baisse de la part relative de l'alimentaire dans les budgets des ménages.

Ensuite, l'essor inéluctable de la grande distribution constituera une contrainte supplémentaire pour les entreprises de PLF: un groupe algérien a annoncé un programme de construction de 10 hypermarchés et de 150 supermarchés sur une période de 15 ans et pour un coût total avoisinant un milliard de dollars ${ }^{17}$. Cette contrainte aval, encore absente en Algérie, n'est pas sans avoir des effets sur le mode et les coûts de distribution des PLF.

16. Office national des statistiques, les dépenses des ménages en 2000 à travers les résultats provisoires de l'enquête consommation, données statistiques, 2002/10, no 352, p. 1-12, ONS, Algérie.

17. Il s'agit du groupe Blanky, d'après les déclarations de son PDG, M. Cherfaoui, dans une interview publiée dans El Watan Economie du 13-19 juin 2005, pages 8-9. 
Enfin, l'installation d'autres firmes agroalimentaires multinationales pourrait avoir des effets importants sur la structure du marché. Ainsi, Nestlé, qui s'est installée en Algérie dans les secteurs des eaux minérales et du lait en poudre avec un partenaire local, pourrait se diversifier dans les produits laitiers frais.

\section{Conclusion}

L'objet de cette recherche était l'étude des relations d'alliances entre FMN et PME dans les pays en voie de transition. Nous devions répondre à trois questions relatives aux enjeux pour un groupe local de s'allier via une PME à une firme multinationale. La compréhension de ces enjeux nécessitait l'analyse des motivations stratégiques de la PME, du déroulement des négociation et des résultats obtenus à la fois par rapport aux autres filiales du groupe local, à sa compétitivité au sein de son secteur d'activité et à son fonctionnement.

Deux principales approches théoriques ont été mobilisées: l'analyse stratégique des alliances pour l'étude des motivations (Garette et Dussauge, 1990) et des changements de la structure du marché (Kogut, 1988) et la théorie de l'apprentissage organisationnel pour analyser les transferts de compétences entre les deux partenaires (Hamel, 1991; Ingham, 1994). Les résultats obtenus confirment en partie les apports théoriques présentés dans notre cadre d'analyse.

Concernant les motivations des partenaires, la PME semble privilégier l'apport d'actifs immatériels par la firme multinationale (image de marque, savoir-faire, expertise en gestion et en marketing). Les apports en capitaux ne constituent pas une motivation essentielle pour l'alliance avec une FMN. Cela est confirmé par la plupart des études sur les alliances stratégiques. Dans leur enquête sur les stratégies interentreprises en France, deux auteurs (Tahi et Belahcen, 2000) avaient abouti à la même conclusion. Les apports tangibles viennent en seconde position dans le classement des besoins des PME, y compris l'apport en technologie. Dans le cas de l'accord DDA, la PME semble parfaitement maîtriser les procédés de production. Son principal besoin était lié à la commercialisation du produit et à la maîtrise des techniques marketing.

Dans une optique stratégique, la motivation de la PME semble confirmer l'hypothèse de Kogut (1988) selon laquelle l'alliance traduirait une stratégie défensive d'évitement d'un concurrent potentiel. Ramanantsoa (1993) soutenait qu'un tel comportement pouvait constituer une marque de compétition indirecte à l'égard de concurrents locaux qui auraient pu envisager l'alliance avec le même partenaire. Autrement dit, la PME algérienne se défend contre Danone (dans le cas où cette firme s'installerait seule en Algérie), mais aussi contre ses concurrents directs en les privant de Danone comme partenaire. Cependant, la manœuvre est symétrique pour Danone. 


\section{TABLEAU 6}

\section{Synthèse du diagnostic de l'accord Danone Djurdjura en Algérie}

\begin{tabular}{|c|c|}
\hline $\begin{array}{l}\text { Motivations de la } \\
\text { PME et apports } \\
\text { des partenaires? }\end{array}$ & $\begin{array}{l}\text { Stratégie défensive de la PME (si Danone venait à } \\
\text { s'implanter seul ou avec un autre partenaire). }\end{array}$ \\
\hline des partenaires? & $\begin{array}{l}\text { Absence de difficultés financières pour la PME. } \\
\text { Motivations axées sur l'image de marque de la FMN, transfert } \\
\text { de savoir-faire (expertise en gestion, marketing). } \\
\text { Apports de la PME: } \\
\text { connaissances du marché local (habitudes de consommation, } \\
\text { fournisseurs, sous-traitants et distributeurs locaux). }\end{array}$ \\
\hline $\begin{array}{l}\text { Déroulement } \\
\text { des négociations } \\
\text { et changements } \\
\text { opérés? }\end{array}$ & $\begin{array}{l}\text { Pour la PME, l'accord DDA était la première négociation } \\
\text { internationale. } \\
\text { Recours des deux partenaires à des experts externes: Bureau } \\
\text { conseil Swicorp pour Danone et groupe d'experts pour } \\
\text { Djurdjura. } \\
\text { Évaluation de la situation de Djudjura par un commissaire } \\
\text { aux apports désigné par un tribunal algérien. } \\
\text { Prudence dans la rédaction des statuts de DDA de la part de } \\
\text { la PME. }\end{array}$ \\
\hline Q2 & $\begin{array}{l}\text { Contrôle partagé pour les décisions stratégiques et } \\
\text { opérationnelles importantes. } \\
\text { DDA insérée dans les sociétés consolidées par mise en } \\
\text { équivalence et non par intégration globale. } \\
\text { Clauses sur la cession des actions. }\end{array}$ \\
\hline \multirow{3}{*}{$\begin{array}{l}\text { Résultats obtenus } \\
\text { par la PME } \\
\text { et le groupe } \\
\text { agroalimentaire } \\
\text { algérien Batouche } \\
\text { Effets de l'accord } \\
\text { sur le marché des } \\
\text { produits laitiers } \\
\text { frais } \\
\text { Q3 } a, b \text { et } c\end{array}$} & $\begin{array}{l}\text { Exploitation de l'image de marque Danone. } \\
\text { Transferts de savoir-faire en procédure et contrôle de la } \\
\text { qualité. } \\
\text { Apprentissage de la PME freiné, celui de la FMN accéléré } \\
\text { par le nouvel organigramme. }\end{array}$ \\
\hline & $\begin{array}{l}\text { Investissements importants durant les trois premières années } \\
\text { concernant la production et les capacités de stockage. } \\
\text { Doublement du C.A. entre } 2001 \text { et } 2004 . \\
\text { Lancement de nouveaux produits Danone. } \\
\text { Accroissement des synergies interfiliales au sein du groupe } \\
\text { algérien Batouche. }\end{array}$ \\
\hline & $\begin{array}{l}\text { Nouvelle configuration: nouveaux producteurs. } \\
\text { Reconversion d'autres producteurs. } \\
\text { Arrivée d'autres FMN dans les PLF. } \\
\text { Arrivée d'autres FMN (concurrents potentiels) dans les eaux } \\
\text { minérales. }\end{array}$ \\
\hline
\end{tabular}

L'accord lui permet à la fois de limiter la concurrence en s'alliant au leader (Veron, 1998) mais aussi d'acquérir une part de marché existante qu'il aurait à conquérir s'il venait à s'implanter seul. 
Les résultats obtenus en termes de contrôle et d'autonomie vont dans le sens des hypothèses de la plupart des auteurs (Hamel, Doz et Prahalad, 1989; Ohamé, 1990). Le contrôle du capital à 51 \% ne signifie en aucun cas un contrôle systématique de la gestion (Park et Ungson, 1997). La PME étudiée (DDA) semble avoir pris des précautions dans ce sens en incluant dans les statuts de l'entreprise des clauses pour l'associer à la prise de décision dès que celle-ci est importante (pour un investissement supérieur à l'équivalent de 1000 euros, le conseil d'administration vote à la majorité qualifiée des 4/5 des membres). Même la multinationale (Danone) reconnaît le partage du pouvoir de gestion. Il semblerait ainsi que le contrôle «parental» (selon Killing, cité dans Yan, 1998) soit partagé entre les deux «parents».

Sur le plan managérial, la question la plus délicate reste celle de l'apprentissage organisationnel: deux constats sont à faire. D'abord, la «course à l'apprentissage» peut parfaitement correspondre aux situations de transferts de compétences entre les deux partenaires. Toutefois, la FMN semble avoir pris des dispositions en vue de freiner l'apprentissage de son partenaire et accélérer le sien, et cela, par deux manœuvres stratégiques.

- La première manœuvre est une spécialisation des tâches au profit des cadres détachés de Danone (Mowery, Oxley et Silverman,1996) dans le but d'éloigner le partenaire local des domaines ou les compétences de Danone peuvent être transférables; la direction marketing est assurée par un cadre Danone et a été positionnée à Alger, hors de l'usine DDA; le département R-D a été remanié et obéit directement aux directives du centre de recherche et de développement de Danone pour l'Afrique et le Moyen-Orient implanté à Barcelone.

- La deuxième manœuvre est la désignation de cadres détachés de Danone à la tête des directions stratégiques afin d'accélérer l'apprentissage par le groupe des compétences locales de la PME. Hormis la direction des ressources humaines et la direction technique de l'usine, toutes les autres directions sont contrôlées par Danone. Cette manœuvre a un double objectif: rapprocher les cadres de Danone des centres opérationnels de décision en vue de favoriser leur apprentissage et contrôler la circulation/diffusion de l'information transmise par les autres cadres et membres du personnel ou leur étant destinée. Dans ce sens, l'accord peut aboutir à la «domination du partenaire qui apprend vite» (Hamel, 1991) mais aussi à une dévalorisation de l'apport de la PME locale (Veron, 1998). Celle-ci se retrouvera alors dans une position de divulgation d'information supplémentaire afin de maintenir la dépendance du partenaire étranger (Hamel, Doz et Prahalad, 1989). D’un autre côté, la PME semble avoir bénéficié de l'expérience de Danone dans trois domaines: 
les procédures «qualité» (afin de respecter la charte Danone), la négociation d'accords internationaux et la gestion des conflits dans un contexte international (Ingham, 1994). Ainsi, les cadres de DDA (dont des locaux) ont négocié avec succès en mars 2005 un contrat de commercialisation avec un partenaire libyen (El Neghazii) pour l'exportation de produits Danone à partir de l'Algérie.

Concernant le devenir de cet accord, deux scénarios restent envisageables:

- Un élargissement de l'accord par l'implication du partenaire local Batouche dans l'implantation probable de la FMN en Algérie pour ses autres métiers de base (biscuits et eaux minérales). Le groupe local devrait dans cette perspective envisager de se diversifier et créer ou acquérir les filiales correspondantes.

- Une reprise de l'entité commune par la FMN après l'achèvement de son apprentissage du contexte institutionnel local. Cette reprise se ferait par augmentation de la part du capital et le contrôle du management. Ce scénario, le plus probable, n'est pas sans poser des problèmes au groupe local, sachant que les autres filiales sont les fournisseurs de DDA en emballage pour certaines et assurent la logistique et la commercialisation pour d'autres.

Même si elle ne permet pas de généraliser les résultats obtenus, notre étude de cas a montré que la PME locale pouvait garder une certaine autonomie de décision alors que le «partage de capital» lui était défavorable. Il apparaît ainsi que l'asymétrie de taille de départ ne constitue pas forcément un handicap définitif. Les processus d'apprentissage organisationnel, de valorisation de compétences spécifiques et les mécanismes indirects de contrôle peuvent compenser une taille réduite et augmenter ainsi le pouvoir de négociation de la petite entreprise. Cela est d'autant plus vérifié dans un contexte de PVD où le risque pays est généralement élevé et le recours à un partenaire local - même de manière transitoire - pour la FMN est indispensable à son implantation.

\section{Bibliographie}

Artisien, P.F et P. Buckley (1985), «Joint ventures in Yougoslavia: opportunities and constraints», Journal of International Business Studies, vol. 16, n ${ }^{\circ} 1$, p. 111-135.

Baudry, B. (1997), L'économie des relations interentreprises, Paris, La Découverte, coll. «Repères».

Revue internationale P.M.E., vol. 21, nº 1, 2008 
BeAmish, P.W. (1985), «Joint ventures in developed and developing countries», Columbia Journal of World Business, vol. 18, n 1, p. 13-19.

BEAMISH,P.W. et J.C. BANKs (1987), «Equity joint venture and the theory of multinational enterprise », Journal of International Business Studies, vol. 18, n 2, p. 1-16.

Bleeke, J. et D. ERnst (1991), «The way to win in cross border alliances», Harvard Business Review, vol. 69, nº 6, p. 127-135.

BLODGETT, L.L. (1992), «Factors in the instability of international joint venture: an event history analysis », Strategic Management Journal, vol. 13, n 6, p. 475-481.

BuCKLEy, P.J. et M. CASSON (1996), «An economic model of international joint venture strategy », Journal of International Business Studies, vol. 27, n 5, p. 849-876.

Burgers, W.P., C.L. Hill et W. Chan Kim (1993), «A theory of global strategic alliances: the case of the global auto industry», Strategic Management Journal, vol. 14, n 6, p. 419-432.

Cohen, W.M. et D.A. Levinthal (1990), «A new perspective on learning and innovation», Administrative Science Quarterly, vol. 35, p. 128-152.

Fernandez, G. et A. Noel (1993), «L'alliance stratégique: une réponse des PME à la mondialisation », Cahiers de recherche CETAI, HEC, Montréal.

Fernandez, G. (1993), «Les alliances stratégiques», Cahiers de recherche CETAI, Montréal, HEC, avril.

FrANKo, L.G. (1989), «Use of minority and 50-50 joint ventures by U.S. multinationals during 1970s/ the interaction of the host country policies and corporate strategies », Journal of International Business Studies, vol. 20, n 1, p. 19-40.

Garette, B. (1989), «Actifs spécifiques et coopération: une analyse des stratégies d'alliances », Revue d'Économie industrielle, n 50, p. 15-31.

Garette, B et P. Dussauge (1990), «Les alliances stratégiques: analyse et mode d'emploi », Revue française de gestion, septembre-octobre, p. 4-18.

GERINGER, J.M. et L. HÉBERT (1991), «Measuring performance of international joint ventures », Journal of International Business Studies, vol. 22, n 2, p. 249-263.

GILL, J. et R.J. BUTLER (2003), «Managing instability in cross-cultural joint ventures », Long Range Planning, $\mathrm{n}^{\circ}$ 36, p. 543-563.

Gomes-CAsseres, B. (1987), «Joint venture instability: It's a problem? Journal of International Business Studies, vol. 22, n 2, p. 97-102.

HAMEL, G. (1991), «Competition for competence and inter-partner learning within international strategic alliances », Strategic Management Journal, vol. 12, p. 83-102.

Hamel, G., Y. Doz et C.K. Prahalad (1989), «S'associer avec la concurrence: comment en sortir gagnant?», Harvard l'Expansion, $n^{\circ}$ 54, p. 24-32. Texte original «Collaborate with your competitors and win», Harvard Business Review, janvier-février 1989.

HARRIgAN, K.R. (1988), «Joint ventures and competitive strategy », Strategic Management Journal, vol. 9, p. 141-158. 
Hennart, J.F. (1988), «A transaction costs theory of equity joint ventures », Strategic Management Journal, vol. 9, p. 361-374.

Hennart, J.F. et S. Reddy (1997), «The choice between mergers/acquisitions and joint venture: the case of Japanese investors in the United States», Strategic Management Journal, vol. 18, n 1, p. 1-12.

INGHAM, M. (1994), «L'apprentissage organisationnel dans les organisations », Revue française de gestion, $\mathrm{n}^{\circ}$ 97, janvier-février, p. 105-121.

INKPEN, A.C. et P.W. BEAMisH (1997), «Knowledge bargaining, power and instability of international joint venture», The Academy of Management Review, vol. 22, $\mathrm{n}^{\circ} 1$, p. 177-202.

Jolly, D. (2001), Alliances interentreprises: entre concurrence et coopération, Paris, Vuibert.

KENT, D.H. (1991), «Joint ventures vs non joint ventures : an empirical investigation», Strategic Management Journal, vol. 12, p. 387-393.

Kogut, B. (1988), «Joint ventures: theoretical and empirical perpectives», Strategic Management Journal, vol. 9, p. 319-332.

Kogut, B. (1991), «Joint venture and the option to expand or acquire», Management Science, vol. 37, p. 19-33.

Larsson, R., L. Bengtsson, K. Henriksson et J. Sparkes (1998), «The interoganizational learning dilemma: collective knowledge development in strategic alliances », Organization Science, vol. 9, n 3, p. 285-305.

LEE, C. et P.W. BEAmisH (1995), «The characteristics and performance of Korean joint ventures in the LDC's », Journal of International Business Studies, vol. 26, $\mathrm{n}^{\circ} 3$, p. 637-654.

LI, J. (1995), «Foreign entry and survival : effects of strategic choices on performances in international market», Strategic Management Journal, vol. 16, p. 333-351.

Luo, Y., O. Shenkar et M.K. Nyaw (2001), «A dual parent perspective on control and performance in international joint ventures: lessons from a developing economy», Journal of International Business Studies, vol. 32, nº 1, p. 41-58.

Mowery, D.C., J.E. OxLey et B.S. Silverman (1996), «Strategic alliances and interfirm knowledge transfer», Strategic Management Journal, vol. 17, p. 77-91.

O'DwYer, M. et E. O'FLYNN (2005), «MNC-SME strategic alliances: a model framing knowledge value as the primary predictor of governance modal choice», Journal of International Management, vol. 11, n 3, p. 397- 416.

Онаме́, K. (1990), «Pourquoi les alliances échouent-elles ?», Harvard L’Expansion, $\mathrm{n}^{\circ} 56$, p. $25-44$.

Organisation DE COOPÉRATION ET DE DÉVELOPPEMENT ÉCONOMIQUES - OCDE (2001), $P M E$ et partenariats, document de synthèse, OCDE.

PARK, S.H. et G.R. UNGSON (1997), «The effect of national culture, organizational complementarity and economic motivation on joint venture dissolution», The Academy of Management Journal, vol. 40, n 2, p. 279-307.

Revue internationale P.M.E., vol. 21, nº 1, 2008 
PARK, S.H. et M.V. Russo (1996), «When competition eclipses cooperation: an event history analysis of joint venture», Management Science, vol. 42, $\mathrm{n}^{\circ}$ 6, p. 875-890.

PARkhe, A. (1991), «Interfirm diversity, organizational leanrning and longevity in global strategic alliances », Journal of International Business Studies, vol. 22, $\mathrm{n}^{\circ} 4$, p. 579-601.

Pfeffer, J. et P. Novak (1976), «Joint ventures and interorganizational interdependence», Administrative Science Quarterly, vol. 21, p. 398-418.

Ramanantsoa, B. (1993), «Faut-il craindre les alliances au Japon?», Annales des mines, Gérer et comprendre, mars, p. 59-71.

RASTOIN, J.L. (1994), «L'industrie alimentaire mondiale: vers un oligopole à franges », Revue française d'économie: économie et politiques agricoles dans les pays en transition, juin, p. 113-126

RAstoin, J.L., G. GHersi et S. TozAnLi (2003), «L'émergence du capitalisme agroalimentaire dans les pays méditerranéens», communication à la conférence du FEMISE, Marseille.

Rastoin, J.L. et S. Tozanli (1992), «Les multinationales et le système alimentaire mondial: tendances stratégiques», Économies et sociétés, Série «Développement agroalimentaire », $\mathrm{AG} \mathrm{n}^{\circ}$ 21, juin, p. 137-165.

Sim, A.B. et A. Yunus (1998), «Performance of international joint ventures from developing and developed countries: an empirical study in a developing country context », Journal of World Business, vol. 33, n 4, p. 357-377.

Steensma, H.K. et M.A. Lyles (2000), «Explaining joint venture survival in a transitional economy through social exchange and knowledge based perspective», Strategic Management Journal, vol. 21, nº 8, p. 831-851.

TAHI, S. et M. BeLAHCEN (2000), «Les stratégies interentreprises et le défi de la PME/ PMI: le cas français », Actes du séminaire de recherche du groupe de recherche économique et sociale (GRECOS), Université de Perpignan.

TAllman, S.B. et O. Shenkar (1994), «A managerial decision model of international cooperative venture formation», Journal of International Business Studies, vol. $25, \mathrm{n}^{\circ} 1, \mathrm{p} .91-113$.

VERON, P. (1998), «Concurrents, confrères, partenaires et alliés de l'entreprise», Extrait de M. Giget (dir.), La dynamique stratégique de l'entreprise, Paris, Dunod, p. $157-175$.

YAN, A. (1998), «Structural stability and reconfiguration of international joint ventures », Journal of International Business Studies, vol. 29, n 4, p. 773-795.

YAN, A. et B. GRAY (1994), «Bargaining power, management control and performance in U.S. - China joint ventures », The Academy of Management Journal, vol. 37, p. 1478-1517.

YAN, A. et M. ZENG (1999), «International joint ventures instability: a critique of previous research, a reconceptualisation and directions for future research», Journal of International Business Studies, vol. 30, n 2, p. 397- 414.

Revue internationale P.M.E., vol. 21, nº 1, 2008 